\title{
Anti-NMDA receptor encephalitis: epidemiological differences and common challenges
}

\author{
Mihai Ceanga, Ha-Yeun Chung, Christian Geis \\ Section Translational Neuroimmunology, Department of Neurology, Jena University Hospital, Jena, Germany \\ Correspondence to: Christian Geis, MD. Section Translational Neuroimmunology, Hans-Berger Department for Neurology, Jena University Hospital, \\ Am Klinikum 1, D-07747 Jena, Germany. Email: Christian.Geis@med.uni-jena.de. \\ Provenance and Peer Review: This article was commissioned and reviewed by the Section Editor Dr. Jinming Han (Department of Clinical \\ Neuroscience, Center for Molecular Medicine, Karolinska Institutet, Karolinska University Hospital, Stockholm, Sweden). \\ Comment on: Xu X, Lu Q, Huang Y, et al. Anti-NMDAR encephalitis: A single-center, longitudinal study in China. Neurol Neuroimmunol \\ Neuroinflamm 2020. doi: 10.1212/NXI.0000000000000633.
}

Submitted Jan 14, 2020. Accepted for publication Jan 29, 2020.

doi: $10.21037 /$ atm.2020.02.34

View this article at: http://dx.doi.org/10.21037/atm.2020.02.34

The anti-N-methyl-D-aspartate receptor (antiNMDAR) encephalitis, first described in 2007 (1), is an autoimmune disease mediated by antibodies against the NMDAR in the cerebrospinal fluid (CSF) (2). AntiNMDAR antibodies bind to the NR1 subunit, induce receptor internalization and synaptic dysfunction (2) finally causing complex neuropsychiatric symptoms. In 2016, an international consortium proposed diagnostic criteria for autoimmune encephalitis to improve its clinical recognition (3). Epidemiological investigations found it to be the most common antibody-mediated encephalitis (4). So far, however, most epidemiological and genetic studies have been performed predominantly in a Caucasian population (5). There is only limited available data on differences in patient characteristics and treatment regimens arising from regional, socio-economic, or genetic variations. Against this background, the recent paper by $\mathrm{Xu} e t$ al. adds important information on the clinical and therapeutic particularities in a large cohort of Chinese patients with anti-NMDAR encephalitis to date (6).

In a single-center, prospective study $\mathrm{Xu}$ et al. enrolled a remarkable number of 220 confirmed cases of antiNMDAR encephalitis between 2011 and 2017, representing the largest cohort of patients described in China so far. Comprehensive clinical characteristics, imaging, laboratory, and electrodiagnostic results, as well as treatment regimens and clinical outcomes were summarized. Most patients were young females and the most common initial clinical presentations were psychosis and seizures, consistent with previous findings in western countries (2). The rate of patients experiencing relapses (17.3\% in the present cohort) was also within a similar range as compared to $12 \%$ in the cohort reported by Titulaer et al. (7) and 20-24\% reported by others $(8,9)$.

However, memory deficits, movement disorders, speech disturbances, and central hypoventilation were less frequently reported as compared to other published cohorts $(7,10)$. This might be relevant, since movement disorders as well as central hypoventilation and ICU admission were found to be significantly associated with a poor functional status (10). Another study on a Chinese cohort of antiNMDAR encephalitis patients as well as a cohort of Korean patients (11) similarly reported a lower incidence of these symptoms (12). The incidence of tumors (ovarian teratomas in all but one case), was similar to previously reported values of around $20-40 \%$, albeit at the lower margin. Differences in the tumor screening process (both screening modality and re-screening frequency) could account for some of these variations. Further diagnostic workup revealed the expected changes on MRI scans in about $30 \%$ of cases, although abnormalities vary during disease course (2), and the authors only specified that the studies were performed "at onset" of disease. It is remarkable that the rate of CSFpositive/seronegative patients (28.6\%) was higher, whereas the incidence of CSF positive oligoclonal bands [25\% $v s$. $>50 \%$ in $(2,13)]$ as well as the percentage of patients 
with electroencephalogram (EEG) abnormalities was considerably lower $[51.4 \% v s .>80 \%$ in (2)] as compared to previous cohorts. A normal EEG recording in early stages (14), as well as a seronegative status (15) were found to correlate with a milder clinical course and a more positive clinical outcome. These differences, together with the lower incidence of some clinical manifestations of anti-NMDAR encephalitis could, if confirmed, suggest a particular manifestation and disease course in the Asian population of patients.

While data presented by $\mathrm{Xu}$ et al. clearly demonstrate an increase in awareness of NMDAR encephalitis manifested as an increase in the number of newly diagnosed cases between 2011 and 2017, the absolute number of misdiagnosed cases fluctuated at about constant values. This again underscores the difficulties and obstacles encountered in reaching a correct diagnosis in a complex disease with multidisciplinary involvement.

The most obvious differences to previously published data emerge from the treatment decisions in patients with NMDAR encephalitis in this cohort. As such, there is a discrepancy between the numbers of ICU admissions within this study and other reports. In previous studies performed in Western countries ICU admission is $>70 \%$ (7), whereas in this study only $51.1 \%$ of patients with a modified Rankin Scale $(\mathrm{mRS})>4$ were admitted to the ICU. Autonomic dysfunction, which was reported at a lower incidence by $\mathrm{Xu}$ et al., is an important indication for intensive care treatment and was also found to be a major risk factor for poor outcome in autoimmune encephalitis (16). Therefore, intrinsic patient characteristics may also have played a role in the lower percentage of patients treated on the ICU. Alternatively, earlier diagnosis due to increased awareness as compared to former patient cohorts and thus earlier initiation of treatment might contribute to the impression of less severely ill patients needing ICU treatment in this cohort.

Moreover, the treatment strategy was particularly different from previous cohorts and current practice in some other centers $(2,4,7)$ with low use of second-line therapy consisting of rituximab and cyclophosphamide, together in only $7.3 \%$ of patients. Instead, first line therapy consisting of glucocorticoids, intravenous immunoglobulins (IVIG), and plasma exchange (PE) was applied alone, in combination, and repetitively in severe cases. Regarding this first line therapy, it is evident that IVIG was intensively used (90.5\% of patients), whereas PE only to a very low extent (3.2\%). Mycophenolate mofetil and azathioprine were most often used as a long-term immunotherapy. As the authors point out, these differences are most likely based on the limited resources and financial concerns of the patients and their families.

However, despite differences in treatment strategies, the reported clinical outcome was particularly good, with $92.7 \%$ of patients reaching a good clinical outcome, defined as an $\mathrm{mRS} \leq 2$. These results may lead clinicians to question the potential benefit of early and consequent second-line therapy after initial therapy using glucocorticoids, IVIG, and PE. However, while $\mathrm{Xu}$ et al. bring these important and needed new data in a dynamic field, there is some information missing that would have been important to complement and to better understand the results and their implications. Related to the favorable outcome it would be important to know how long severely afflicted patients need to achieve an outcome of $\mathrm{mRS} \leq 2$ without early initiation of second-line therapy, e.g., rituximab. This is of importance as these patients are at high risk for developing complications in the severe phase of the disease, e.g., during intensive care (16). Risk factors other than tumors need to be assessed in more depth in future studies, including previous herpesvirus infection but also possible genetic factors.

Moreover, the excellent outcome on the mRS scale may obscure ongoing deficits, in particular neurocognitive dysfunction as the mRS scale is inappropriate to depict the complete clinical syndrome of a complex neuropsychiatric disorder. Studies in both paediatric (17) and adult (18) patients have emphasized the complexity of neuropsychological deficits as well as their refractory nature leading to severe limitation of patients' quality of life. Regarding the symptoms of initial presentation, and more importantly with respect to the long-term outcome, a more formal description of neuropsychological deficits should be implemented. This again proves to be a common challenge, as exemplified by a recent review of cognitive outcomes after autoimmune encephalitis, in which 546 publications out of 975 had to be excluded due to insufficient description of the neurocognitive status (19). Reasons for incomplete neuropsychological evaluation are manifold and comprise comatose patients in the most severe phase of disease as well as confounding factors, e.g., ongoing psychotic behavior and selection of the appropriate neuropsychological test battery. While severe memory impairment can have an imposing and easily recognizable bedside presentation, less severe deficits may initially go unnoticed, making even a basic but standardized, comprehensive and repeated neuropsychological examination necessary. This should be initiated as soon as patients recover from the very acute 
phase. Although none of the neuropsychological testing procedures is validated for the anti-NMDAR encephalitis, a combination of the Montreal Cognitive Assessment (MoCA) with the Rey Auditory Verbal Learning Test (RAVLT) and Neuropsychiatric Inventory (NPI) might be a comprehensive approach. Hopefully, this might allow a better interstudy evaluation of neuropsychological symptoms and comparison of different treatment strategies.

Although NMDA receptor encephalitis gained more attention in the last decade, it remains a major challenge to physicians worldwide to investigate the disease due to limited patient numbers. With an estimated incidence of 1.5 per million population per year, anti-NMDAR encephalitis is a rare disorder (2). Due to the limited patient number, the importance of networking to increase our knowledge concerning pathophysiological mechanisms and therapeutic strategies is critical for clinical research. We agree with $\mathrm{Xu}$ et al. that further prospective multicenter studies are warranted to investigate the efficacy of acute and long-term immunotherapy. Moreover, since there is no specific therapy so far and recovery from NMDAR encephalitis is often delayed despite current treatment approaches, prospective controlled clinical studies are needed to investigate innovative regimens of immunotherapy and eventually also targeted therapy beyond and in addition to immunotherapy. These approaches of e.g., NMDAR modulation $(20,21)$ are currently evaluated in preclinical research and may hopefully develop to clinical options in the future.

\section{Acknowledgments}

Funding: This work was supported by the Schilling Foundation and the German Research Council, DFG RU 3004 (to CG).

\section{Footnote}

Conflicts of Interest: All authors have completed the ICMJE uniform disclosure form (available at http://dx.doi. org/10.21037/atm.2020.02.34). The authors have no conflicts of interest to declare.

Ethical Statement: The authors are accountable for all aspects of the work in ensuring that questions related to the accuracy or integrity of any part of the work are appropriately investigated and resolved.

Open Access Statement: This is an Open Access article distributed in accordance with the Creative Commons Attribution-NonCommercial-NoDerivs 4.0 International License (CC BY-NC-ND 4.0), which permits the noncommercial replication and distribution of the article with the strict proviso that no changes or edits are made and the original work is properly cited (including links to both the formal publication through the relevant DOI and the license). See: https://creativecommons.org/licenses/by-nc-nd/4.0/.

\section{References}

1. Dalmau J, Tuzun E, Wu HY, et al. Paraneoplastic anti-Nmethyl-D-aspartate receptor encephalitis associated with ovarian teratoma. Ann Neurol 2007;61:25-36.

2. Dalmau J, Armangue T, Planaguma J, et al. An update on anti-NMDA receptor encephalitis for neurologists and psychiatrists: mechanisms and models. Lancet Neurol 2019;18:1045-57.

3. Graus F, Titulaer MJ, Balu R, et al. A clinical approach to diagnosis of autoimmune encephalitis. Lancet Neurol 2016;15:391-404.

4. Dalmau J, Graus F. Antibody-Mediated Encephalitis. N Engl J Med 2018;378:840-51.

5. Mueller SH, Farber A, Pruss H, et al. Genetic predisposition in anti-LGI1 and anti-NMDA receptor encephalitis. Ann Neurol 2018;83:863-9.

6. Xu X, Lu Q, Huang Y, et al. Anti-NMDAR encephalitis: A single-center, longitudinal study in China. Neurol Neuroimmunol Neuroinflamm 2020. doi: 10.1212/ NXI.0000000000000633.

7. Titulaer MJ, McCracken L, Gabilondo I, et al. Treatment and prognostic factors for long-term outcome in patients with anti-NMDA receptor encephalitis: an observational cohort study. Lancet Neurol 2013;12:157-65.

8. Irani SR, Bera K, Waters P, et al. N-methyl-D-aspartate antibody encephalitis: temporal progression of clinical and paraclinical observations in a predominantly non-paraneoplastic disorder of both sexes. Brain 2010;133:1655-67.

9. Gabilondo I, Saiz A, Galan L, et al. Analysis of relapses in anti-NMDAR encephalitis. Neurology 2011;77:996-9.

10. Balu R, McCracken L, Lancaster E, et al. A score that predicts 1-year functional status in patients with antiNMDA receptor encephalitis. Neurology 2019;92:e244-52.

11. Lim JA, Lee ST, Jung KH, et al. Anti-N-methyl-daspartate receptor encephalitis in Korea: clinical features, treatment, and outcome. J Clin Neurol 2014;10:157-61.

12. Zhang Y, Liu G, Jiang M, et al. Clinical Characteristics and 
Prognosis of Severe Anti-N-methyl-D-aspartate Receptor Encephalitis Patients. Neurocrit Care 2018;29:264-72.

13. Blinder T, Lewerenz J. Cerebrospinal Fluid Findings in Patients With Autoimmune Encephalitis-A Systematic Analysis. Front Neurol 2019;10:804.

14. Sonderen AV, Arends S, Tavy DLJ, et al. Predictive value of electroencephalography in anti-NMDA receptor encephalitis. J Neurol Neurosurg Psychiatry 2018;89:1101-6.

15. Guasp M, Modena Y, Armangue T, et al. Clinical features of seronegative, but CSF antibody-positive, antiNMDA receptor encephalitis. Neurol Neuroimmunol Neuroinflamm 2020. DOI: https://doi.org/10.1212/ NXI.0000000000000659.

16. Schubert J, Bramer D, Huttner HB, et al. Management and prognostic markers in patients with autoimmune encephalitis requiring ICU treatment. Neurol Neuroimmunol Neuroinflamm 2018;6:e514.

17. de Bruijn M, Aarsen FK, van Oosterhout MP, et al. Long- term neuropsychological outcome following pediatric antiNMDAR encephalitis. Neurology 2018;90:e1997-2005.

18. Al-Diwani A, Handel A, Townsend L, et al. The psychopathology of NMDAR-antibody encephalitis in adults: a systematic review and phenotypic analysis of individual patient data. Lancet Psychiatry 2019;6:235-46.

19. McKeon GL, Robinson GA, Ryan AE, et al. Cognitive outcomes following anti-N-methyl-D-aspartate receptor encephalitis: A systematic review. J Clin Exp Neuropsychol 2018;40:234-52.

20. Warikoo N, Brunwasser SJ, Benz A, et al. Positive Allosteric Modulation as a Potential Therapeutic Strategy in Anti-NMDA Receptor Encephalitis. J Neurosci 2018;38:3218-29.

21. Planaguma J, Haselmann H, Mannara F, et al. Ephrin-B2 prevents $\mathrm{N}$-methyl-D-aspartate receptor antibody effects on memory and neuroplasticity. Ann Neurol 2016;80:388-400.
Cite this article as: Ceanga M, Chung HY, Geis C. AntiNMDA receptor encephalitis: epidemiological differences and common challenges. Ann Transl Med 2020;8(11):716. doi: 10.21037/atm.2020.02.34 